Classification

Physics Abstracts

$6810-6890-6110$

\title{
Structural properties and elasticity of amphiphilics on water
}

\author{
J Dallant $\left({ }^{1}\right)$, L Bosio $\left({ }^{2}\right)$, B Harzallah $\left({ }^{2,},{ }^{3}\right)$ and J. J. Benattar $\left({ }^{1}\right)$
}

(1) Service de Physique du Solide et de Résonance Magnétıque, Direction de la Physique, Centre d'Etudes Nucléaires de Saclay, 91191 Gif-sur-Yvette Cedex, France

$\left({ }^{2}\right)$ Laboratoire Physique des Liquides et Electrochımı, ESPCI, 10 rue Vauquelın, 75321 Parıs Cedex 05, France

$\left({ }^{3}\right)$ Département de Physıque, Faculté des sciences, 5000 Monastır, Tunısıa

(Recelved 19 September 1990, accepted in final form 8 November 1990)

\begin{abstract}
Résumé. - On a étudié par réflectıvité des rayons $\mathrm{X}$ les monocouches d'amphıphıles à l'ınterface eau-air Cette technıque permet une détermınation précıse et indépendante des épaisseurs (par la mesure de franges d'égale inclınaıson) aınsı que des densıtés et des rugosıtés interfacıales (1l s'agıt d'une mesure absolue), cecı permet de remonter à un profil complet de densité électronıque La rugosité, quı est duc $1 c 1$ aux ondes capillaires excitées thermiquement est donc reliée à la dynamıque de l'interface, quı dépend des constantes élastıques de la monocouche Les diagrammes de phases des acides gras en $\mathrm{C}_{15}, \mathrm{C}_{21}$ et $\mathrm{C}_{29}$ ainsı que du phospholipide L- $\alpha$-DPPC ont été explorés et une analyse des transitions de phase a été réalısée Du point de vue structural, une caractérıstıque générale de cette classe de composés adsorbés à la surface de l'eau est que la densité du milıeu alıphatıque est constante dans les phases lıquide et solıde Il s'ensuit que les courbes pression de surface-épaisseur du film reflètent les courbes pression surface En particulier la transition liquide-expansé liquide-condensé est révélée par un accroissement brusque de l'épaisseur du milıu ahphatıque. Les résultats suggèrent que cette transition s'accompagne de changements de conformation des chaînes De même la décroıssance de l'aıre par molécule dans la phase liquide-condensé se traduit-elle par une dimınution de l'inclınaison des molécules On montre aussi que la structure de cette phase est indépendante de la température et de la nature du groupe polaire Une transition vers une multıcouche est observée dans le cas des chaînes les plus longues On montre que la dynamique de l'interface est compatible dans les phases liquides avec un modèle simple d'ondes capillaires, sans paramètre ajustable La rugosité du film chute à la transition vers le solıde, ce qu révèle une grande résistance à la courbure Les valeurs élevées du module de nigidité que l'on obtıent (de l'ordre de $100 k_{\mathrm{B}} T$ ) sont sensibles à la longueur des chaînes carbonées et peuvent s'interpréter en fonction d'un modèle simple. La diffusion des rayons X par les ondes capillaires est détaillée dans le cas d'une interface rigide tel qu'une monocouche sur l'eau
\end{abstract}

\footnotetext{
Abstract. - Monolayers of amphiphilic molecules adsorbed at the arr-water interface have been studied using X-ray reflectivity This technique allows an accurate and independent determination of thicknesses (by the measurement of fringes of equal inclination) as well as densities and roughnesses (since this is an absolute intensity measurement), therefore a complete electron density profile can be obtained The surface roughness is due to thermally excited capillary waves, and is thus related to the dynamics of the interface, which are conditionned by the elastic constants of the layer The phase diagrams of the $C_{15}, C_{21}$ and $C_{29}$ fatty acids and of the
} 
phospholıpid L- $\alpha$-DPPC have been investigated using this method, and an analysis of the phase transitions has been performed. From the structural point of view, a general feature of this class of compounds adsorbed on water is an unchanging aliphatic medium density within the liquid and solid phases. If follows that the pressure $v s$ film thickness curves mimic the pressure area curves In particular evidence for the liquid-expanded to liquid-condensed transition is given by an abrupt increase of the alıphatic medium thickness. The data suggest that this transition should involve conformational changes Furthermore the decrease in molecular area within the liquid condensed phase is revealed by a decreasing molecular tilt angle The structure of this phase is also shown to be independent of the temperature and of the nature of the polar head-group A transition to a multilayer structure is observed with the longest chain compound We show that the dynamics of the interface are consistent in the hquid phases with a simple capillary wave model, with-no adjustable parameter $\mathrm{A}$ strong drop in the roughness at the transition to the solid state reveals the onset of a rigidity against bending The high values of the rigidity modulus obtained (on the order of $100 k_{\mathrm{B}} T$ ) are sensitive to the aliphatic chain length and can be interpreted using a simple model for the elastic behavior of the solid monolayer The scattering of $\mathrm{X}$-rays by capillary waves in detailed for a ngid interface such as a monolayer

\section{Introduction.}

Monolayers of amphiphilic molecules adsorbed at the a1r-water interface have drawn considerable attention [1-7] and the interest in these systems has been recently renewed by the development of new non-perturbative techniques of investigation of the surface of liquids One of the most fascinating aspects exhibited by these two-dimensional systems is the great variety of phase transitions [4-7] revealed by kinks in the isotherms $(\Pi, \mathcal{A})$, where $\Pi$ is the surface pressure $\left(\Pi=\gamma_{\text {water }}-\gamma\right)$ and $\mathfrak{A}$ the molecular area

From a gaseous-like state, condensation can occur to the so-called hquid expanded (LE in the nomenclature of Adam, $\mathrm{L}_{1}$ according to that of Harkins) and then to the more condensed and less compressible liquid-condensed $\left(\mathrm{LC}\right.$ or $\left.\mathrm{L}_{2}\right)$ phase, or directly to the LC phase at temperatures under that of the triple point $T\left(\mathrm{~F}_{1} \mathrm{~g} 1\right)$. The question of the order of this LE/LC transition has been most controversial. Mechanisms like reduced cooperavity of the transition [8] or interactions between domains [9] have been invoked to explain the finite slope of the isotherm in the coexistence region (which has even been considered as a distinct « intermediate " phase [2]). Pallas and Pethica [10] have shown, however, that one can obtain flat isotherms using carefully purified materials, and it is now well recognized that this transition is of first order. Further evidence is the existence of domains at the transition, which has been demonstrated using light scattering [11] (fluorescence microscopy [12] requires the introduction of fluorophores that may modify the properties of the monolayer).

On further compression of long-chain compound monolayers, or at lower temperatures (1ncreasing the chain length by one alkyl group is equivalent to lowering the temperature by about $6^{\circ} \mathrm{C}$ [3]), a variety of condensed phases can be attained Following Harkins [2], these are referred as liquid condensed $\left(\mathrm{L}_{2}^{\prime}\right.$ and $\left.\mathrm{L}_{2}^{\prime \prime}\right)$, and solid phases, characterized by their extremely lo,w compressibility and their stıffness (LS « superliquid » [sic], S « solid", and CS "condensed solid »). The order of the liquid-condensed to solid transition depends on which of the $\mathrm{L}_{2}, \mathrm{~L}_{2}^{\prime}$, LS or $\mathrm{S}$ phases are implicated. A schematic phase diagram for fatty acids or esters for example is given in figure 1 [4-6, 13]. Note that each phase is not necessarily obtained with a given compound (especially with the shorter chain lengths) and that, conversely, more than one phase of each kind is obtainable with a given compound [6].

The properties of such films have been intensively investigated by various techniques mechanical measurements allow the determination of elastic constants of the monolayers such as shear modulı [14] and their viscoelastic behavior can be investigated by means of light 


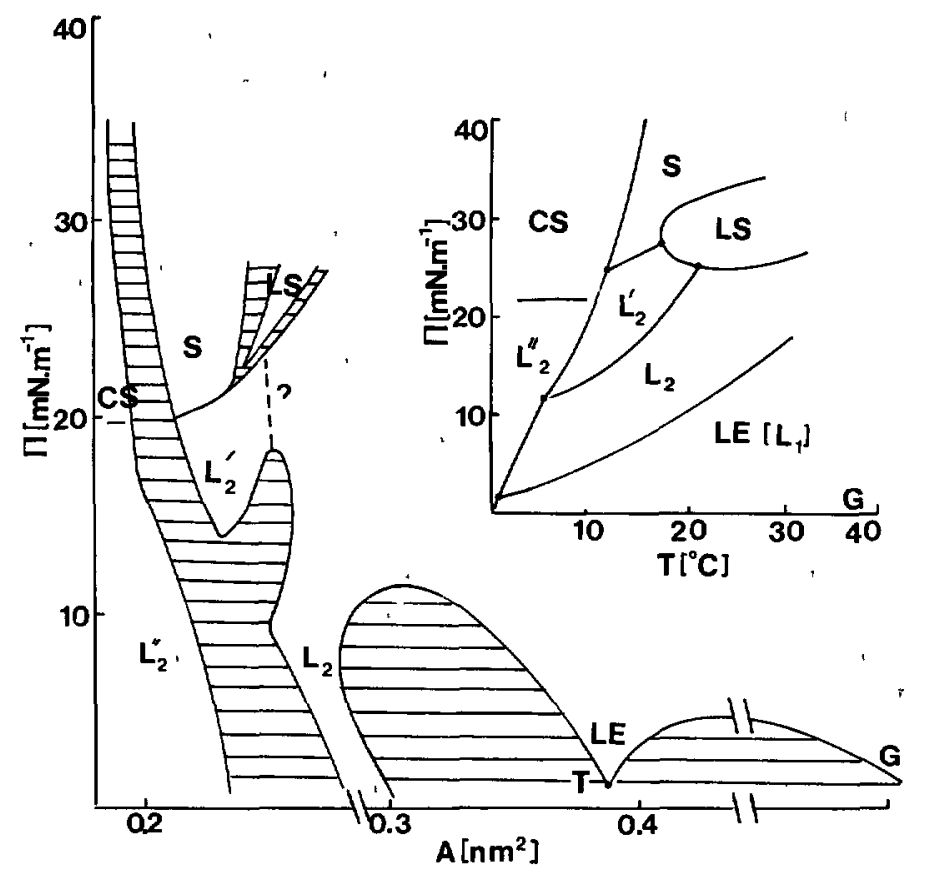

Fig 1 - Schematic phase diagram of fatty-acid monolayers according to reference $[2,4-6,13]$; striped areas are coexistence regions. Insert $\cdot$ temperature-pressure section

scattering experiments [15-17]. The orientation of the polarisible $\mathrm{C}-\mathrm{OH}$ bonds has" been determined by' optical second harmonic generation [18] and information about the surface density of dipoles can be obtained by surface potential measurements [1, 19] More recently, the morphology of coexisting domains in first-order transitions has been investigated by fluorescence microscopy $[12,19]$. A decisive advance has been the determination of the in-plane order of condensed films by grazing incidence X-ray diffraction [20-22] Lin et al have recently investigated the phase diagram of heneicosanoic acid up to a temperature of $8.5^{\circ} \mathrm{C}$, and characterized its different phases [22] This technique is complemented by X-ray [23-25] or neutron [26] reflectivity experiments

For this study we have used X-ray reflectivity which allows the determination of the electron density profile normal to the surface, $1 \mathrm{e}$. an accurate determination of thicknesses, of roughnesses and (since this is an absolute measurement) of densities. Both the liquidexpanded to liquid-condensed and liquid-condensed to solid transitions have been considered (E1ther the Adam or the Harkins nomenclature will be used in the following, depending on which is the more appropriate, with no assumption concerning the nature of the phases) After a presentation of the X-ray reflectivity technique and of the experimental details, a third part of the paper will be first devoted to the discusion of the structural transformations of monolayers, and then to their effect on the dynamics of the interface The scattering of Xrays by capillary waves is 'examined from their correlation function for a rigid interface in the appendix

\section{X-ray reflectivity.}

11 X-RAY REFLECTIVITY AND THE DETERMINATION OF ELECTRON DENSITY PROFILES. For $\mathrm{X}$-ray wavelengths, the refractive index of matter $n$ is given by $n=1-\delta-\imath \beta$ The 
imaginary component $\beta$ is proportional to the linear absorption coefficient, and $\delta$ to the electron density of the material [27] For water and the CuK $\alpha_{1}$ radiation $(\lambda=015405 \mathrm{~nm})$ $\beta=0.0126 \times 10^{-6}$ and $\delta=3.56 \times 10^{-6}$. Since the frequency of the electromagnetic radiation is much larger than the characteristic atomic transition frequencies of the elements composing the organic compounds and water $(\mathrm{H}, \mathrm{C}, \mathrm{O}, .$.$) , the electrons can be considered as free$ particles Therefore, the refractive index is determined at a local scale [28] and the accuracy of the electron density determination only depends upon the resolution of the experiment For $\mathrm{X}$-ray reflectivity, which consists in measuring the intensity of the beam reflected at an interface as a function of the angle of incidence, the wave-vector transfer $q$ is perpendicular to the surface of the substrate $\left(\mathbf{q}=q_{z} \mathbf{z}, q_{z}=4 \pi \sin \theta / \lambda\right.$, where $\theta$ is the angle of incidence). Hence, in the plane of the interface, the average is taken over the area of coherence of the beam [29] (here typically $\approx 0.5 \mu \mathrm{m}^{2}$ ), and one obtains the mean characteristics of the surface averaged over distances up to a few tens of $\mu \mathrm{m}$ Along $z$, one gets very accurate information about the projected electron density $\rho(z)$ (at a scale at most $\approx \pi / q_{\max } \approx 0.35 \mathrm{~nm}$ since the larger incident angle is $\theta_{\max } \approx 50 \mathrm{mrad}$ ) Often one can obtain even more precise determ1nations by the use of a priort, generally chemical, constraints. It is shown in the appendix that even a rough interface is adequately described for specular reflectivity by an average electron density profile $\langle\rho(z)\rangle_{x, y}$

Since the real part of the index is less than 1 , total external reflection occurs for grazing angles below a critical angle $\theta_{\mathrm{c}}=\sqrt{2 \delta}\left(\theta_{\mathrm{c}}=267 \mathrm{mrad}\right.$ for water $)$ For a single ideal diopter the reflected intensity follows the Fresnel law $\left(R_{\mathrm{F}}(\theta) \approx\left(\theta / 2 \theta_{\mathrm{c}}\right)^{4} ; \theta \gg \theta_{\mathrm{c}}\right)$. Let us note that, since $r_{\mathrm{p}} / r_{\mathrm{s}} \approx 1+4 \theta^{2}$ (where $r_{\mathrm{s}}$ and $r_{\mathrm{p}}$ are the reflection coefficients for $\mathrm{s}$ and $\mathrm{p}$ polarizations), polarization effects may be neglected.

If the angle of incidence $\theta$ is sufficiently greater than $\theta_{c}$ and multiple scattering effects may be neglected, the equivalent of the first Born approximation in quantum theory becomes very accurate [30] and provides a transparent mean to understand the reflectivity curves. In this approximation, the reflectivity averaged over the coherence of the beam is given by $[31,32]$

$$
\left\langle R\left(q_{z}\right)\right\rangle=R_{\mathrm{F}}\left(q_{z}\right)\left\langle\left|\frac{1}{\rho_{\mathrm{s}}} \int \mathrm{d} z \mathrm{e}^{\imath q_{z} z} \frac{\partial \rho(z)}{\partial z}\right|^{2}\right\rangle
$$

where $\rho_{\mathrm{s}}$ is the electron density of the substrate, $R_{\mathrm{F}}\left(q_{z}\right)$ is the Fresnel reflectivity of the substrate surface 1.e the reflectivity of a perfect diopter having the bulk density of the substrate. Equation (1) is subject to two kınds of limitatıons : Firstly, the Born approximation requires small scattering cross sections (and this is verified here for $\theta \geqslant 3 \theta_{\mathrm{c}}$ ), secondly, the density gradients must be small as - compared to the wavelength $(1 / k \rho)(\partial \rho / \partial z) \approx(2 \pi)^{-3 / 2}\left(\lambda /\left\langle z^{2}\right\rangle^{1 / 2}\right) \approx 003$ for a roughness $\left.\left\langle z^{2}\right\rangle^{1 / 2}=03 \mathrm{~nm}\right)$.

1.2 X-RAY REFLECTIVITY STUDY OF LANGMUIR FILMS - The shape of the reflectivity curve (Eq (1)) results from interferences between the beams reflected by the surface and different interfaces As in many similar cases, the present system can be described as composed of chemically homogeneous layers, and a model can be constructed by considering these layers as slabs of constant density. Then, one can take into account the roughness of an interface (the standard deviation of its location $\mathbf{z}$ ) by smearing this model through a convolution with a Gaussian function (see the appendix), leading to :

$$
\left\langle R\left(q_{z}\right)\right\rangle=R_{\mathrm{F}}\left(q_{z}\right)\left\langle\mid \frac{1}{\rho_{\mathrm{s}}} \sum_{i=1}^{n}\left(\rho_{l}-\rho_{\imath-1}\right) \mathrm{e}^{i q_{z} z_{l}} \mathrm{e}^{-1 /\left.2 q_{z}^{2} \sigma_{l}^{2}\right|^{2}}\right\rangle
$$

where $z_{l}$ is the location of the $(l-1 / l)$ interface, $\rho_{l}$ the electron density of the $l$-th slab, and $\sigma_{l}$ the roughness of the $(i-1 / l)$ interface For a liquid interface, this roughness is due to 
thermally excited capillary waves With a surface tension $\gamma$, a bending rigidity $K$, and a halfwidth at half maximum $\Delta q_{x}$ of the resolution function of the diffractometer along the $x$-axis, one obtains (see the appendix)

$$
\sigma^{2}=\frac{k_{\mathrm{B}} T}{4 \pi \gamma}\left[\log \left(\frac{\gamma}{K \Delta q_{x}^{2}}\right)-\left(\gamma_{\mathrm{E}}+\log 2\right)\right]
$$

where $\gamma_{E}$ is Euler's constant The reflectivity curve, calculated from (2), is fitted to the experimental data in order to obtain the characteristics of the monolayer Since $\sigma . \Delta q_{z} \ll 1$, convolution with the experimental resolution does not change the results in the present case, and this has been verified. It should be emphasized that when building such an electron density model, one must take into account the natural separation of the amphiphilic molecules into polar heads and aliphatic chains. An attempt to fit using a single lamina model fails, leading to a much too broad interference peak. It 1s, nevertheless, generally meaningless to assign different roughnesses at the air/chain, chain/head and head/water interfaces (the fit leads to very close results, or non significative variations)

The well defined interference peaks observed with monolayers yield separate information about the structure of the interface and the roughness. Actually, whereas the roughness causes a continuous attenuation of the reflected intensity, certain regions of the curve are highly sensitive to a given parameter of the fit the overall thickness of the amphiphilic monolayer is given by the location of the first destructive interference, whose contrast is mainly determined by the surface density of the polar heads. Furthermore the part of the curve recorded at grazing angles below this first intensity minimum is related to the density of the aliphatic chains.

From the complete electron density profile, one can calculate surface excess quantities $\Gamma$ [33]. As an example, the number of electrons in the aliphatic medium per unit surface is $\Gamma_{\mathrm{c}}=\rho_{\mathrm{c}} \ell_{\mathrm{c}}$ where $\rho_{\mathrm{c}}$ and $\ell_{\mathrm{c}}$ are the electron density and the length of the tails determined experimentally The quantity $\Gamma$ enlightens another aspect of the problem : the total amount of matter in a given lamina is determined, whereas densities, thicknesses, and roughnesses give information about the distribution of this matter. The molecular area can easily be obtained from $\Gamma_{\mathrm{c}}: \mathcal{A}=n_{\mathrm{c}} / \Gamma_{\mathrm{c}}$ where $n_{\mathrm{c}}$ is the number of electrons per chan For very thin layers ( $\leqslant 0.35 \mathrm{~nm}$ ) such as the headgroup region, direct information can be obtained only about $\Gamma$, but not about $\rho$ or $\ell$.

A last important point to be discussed is that of the analysis of the experimental results when domains of different phases coexist in the monolayer. For example, it has been established [11] that, in the LE/LC coexistence region, LC domains of typical size $0.4 \mathrm{~mm}$ are embedded in the LE phase, as expected for a first-order transition [10]. According to Grundy et al., [34], if this size is smaller than the coherence area of the beam ( $\approx 04 \mu \mathrm{m} \times 40 \mathrm{~nm}$ ), the film can be considered as homogeneous Otherwise, the reflectivity is the average of that of the two phases Practically, the difference is extremely small in all the cases encountered in this work and prevents us from distınguishıng between the two possibilities, but justifies our following simplification treating the film as homogeneous

\section{Experimental details.}

2.1 REFLECTOMETER FOR LIQUIDS AND LANGMUIR TROUGH. - The diffractometer for liquids has been described elsewhere [35] The $\operatorname{CuK} \alpha_{1}$ radiation is selected using a flat $\mathrm{LiF}(200)$ monochromator and a small divergence slit $\mathrm{S}_{\mathrm{D}}(50 \mu \mathrm{m})$ followed by an antidiffusion slit $\mathrm{S}_{\mathrm{AD}}(70 \mu \mathrm{m})$. The divergence is low $(023 \mathrm{mrad})$. The intensity of the monochromated beam is monitored by an ionization chamber $I_{C}$ and that of the reflected beam is detected by a 
scintillation counter placed behind the analysis slit $\mathrm{S}_{\mathrm{A}}\left(\Delta_{x} \times \Delta_{y}=50 \mu \mathrm{m} \times 10 \mathrm{~mm}\right)$ The source-to-sample distance is $40 \mathrm{~cm}$, and the sample-to-detector distance $30 \mathrm{~cm}$. With those dimensions, the HWHM of the resolution function is $\Delta q_{x}=1.2 \times 10^{5} \mathrm{~m}^{-1}$, at $\theta=30 \mathrm{mrad}$, along $x$, and $\Delta q_{y}=68 \times 10^{8} \mathrm{~m}^{-1}$ along $y$

A reflectivity curve is recorded by scanning across the reflected beam at each incident angle to determine the specular reflectivity and the diffuse background Using a conventional X-ray tube $(15 \mathrm{~kW})$, we can measure reflectivities $R\left(q_{z}\right)$ down to $10^{-7}$.

Our Langmuir trough [25], made of Teflon, has been designed to allow grazing incidence X-ray experiments Most important in this case is to adjust the surface level, this is achieved by displacement of an auxilliary reservoir to withın $\pm 5 \mu \mathrm{m}$.

The area is fixed by means of a Teflon ribbon whose ends are pinned at two opposite corners of the trough, thus avoiding losses [3] The surface pressure (measured using a filterpaper Wilhelmy balance [36]) can be used to control the area. In order to avold surface vibrations, the liquid layer was only $3 \mathrm{~mm}$ deep and the experiments were carried out overnight

The trough can be temperature controlled to within $\pm 0.2{ }^{\circ} \mathrm{C}$. The system is enclosed in a humidity saturated box $2 \mathrm{~cm}$ high whose cover can also be heated and is maintained under a slow nitrogen flow.

22 CHEMICAL - Docosanoic acid (behenic acid) $\mathrm{CH}_{3}-\left(\mathrm{CH}_{2}\right)_{20} \mathrm{COOH}$, and triacontanoic acid (melissic acid) $\mathrm{CH}_{3}-\left(\mathrm{CH}_{2}\right)_{28}-\mathrm{COOH}$ were purchased from Fluka (purissimum, for gaz

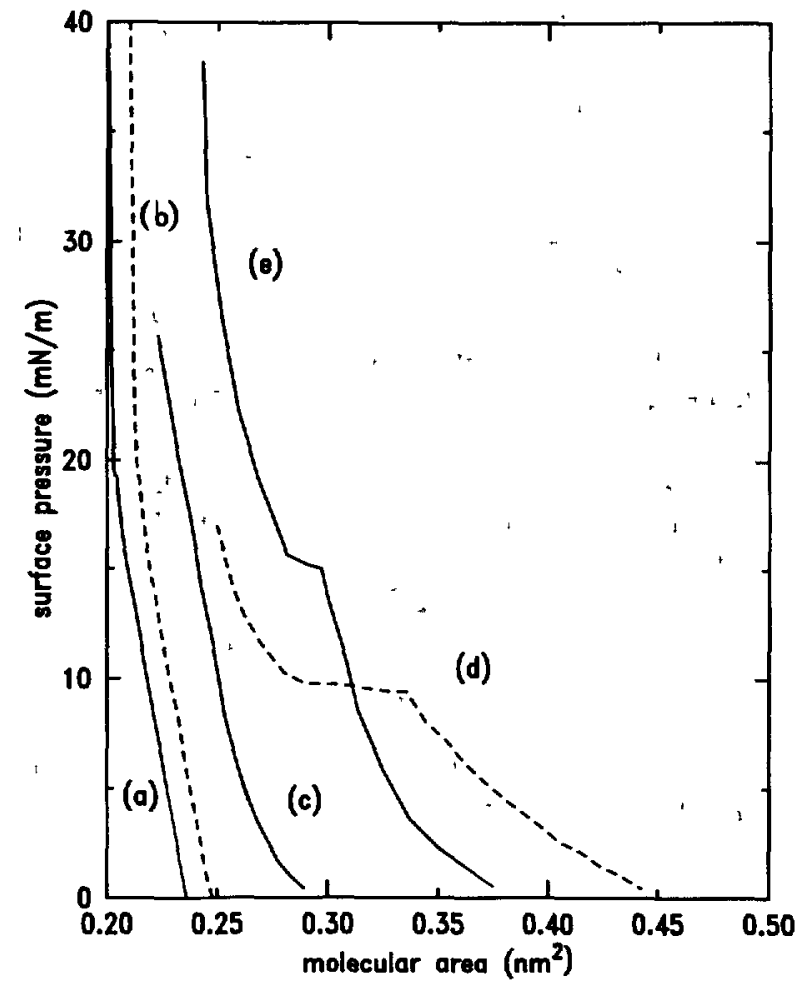

Fig 2. - Measured isotherms of monolayers at the air-water interface, (a) behenic acid $T=23^{\circ} \mathrm{C}$, (b) melissic acid $T=23{ }^{\circ} \mathrm{C}$, (c) palmitic acid $T=22{ }^{\circ} \mathrm{C}$, (d) palmitic acid $T=33{ }^{\circ} \mathrm{C}$, (e) L- $\alpha$-DPPC $T \doteq 23^{\circ} \mathrm{C}$ For clarity each curve has been shifted from the previous by $001 \mathrm{~nm}^{2}$ In the case of DPPC the area per one chain has been reported 
chromatography), and used as obtained Behenic acid was dissolved in chloroform, and melissic acid in a $\mathrm{n}$-hexane/chloroform/toluen mixture The 1sotherm recorded at room temperature $\left(T=23^{\circ} \mathrm{C}\right)$ for behenic acid goes through the $\mathrm{L}_{2}, \mathrm{~L}_{2}^{\prime}, \mathrm{LS}$ and $\mathrm{S}$ phases (Fig. 2) [5] It exhibits a kınk at the $\mathrm{L}_{2}^{\prime} / \mathrm{LS}$ (or liquid condensed to solid) transition

Hexadecanoic acid (palmitic acid) $\mathrm{CH}_{3}-\left(\mathrm{CH}_{2}\right)_{14}-\mathrm{COOH}$ allows both the investigation of the $\mathrm{LC}$ phase at room temperature and the $\mathrm{LE} / \mathrm{LC}$ transition at a higher temperature since the triple point temperature is $29^{\circ} \mathrm{C}$ [10] This sample (Fluka, purissimum, for gaz chromatography, $99.9 \% \mathrm{~min}$.) was further purified by slow recrystallization from a hexane solution The spreading solution was prepared by selectıng a monocrystal, as checked by X-ray diffraction, then dissolved in $\mathrm{n}$-hexane $\left(\sim 5 \mathrm{mg} \mathrm{ml}^{-1}\right)$. The $\mathrm{n}$-hexane used in all these procedures was purified by slow distillation, and this point appeared to be the most important in order to obtain the flat LE/LC coexistence regions of figure $2[10,11]$

The LE, LC and solid phases were studied using L- $\alpha$-dipalmitoylphosphatidylcholıne (DPPC) at room tempetrature L- $\alpha$-DPPC from Sigma was used as obtained, dissolved in chloroform.

Each experiment was performed on a fresh subphase (ultra-pure water from a millipore system) whose $\mathrm{pH}=2$ was adjusted using concentrated $\mathrm{HCl}$. Small volumes $(10 \mu \mathrm{l})$ of the solution were spread at the surface, and, after evaporation of the solvent and very slow compression, the system was left to reach equilibrium The X-ray experiments which lasted $\sim 12 \mathrm{~h}$ long were performed on clearly equilıbrated (but metastable, vide infra) layers [36, 37].

\section{Results and discussion.}

A first step when studying monolayers at the air-water interface 1s, of course, to carefully characterize the bare water surface $[24,38]$. The corresponding reflectivity curve (normalized to $R_{\mathrm{F}}(\theta)$ ) (Fig 3) has a Gaussian shape, and the roughness is $\sigma \approx 0.31 \mathrm{~nm} \pm 001 \mathrm{~nm}$. (The error bars are estımated by assumıng Poisson statistıcs for each independent counting process and according-to the contour $\Delta \chi^{2}=\chi^{2}-\chi_{\min }^{2}=1$ in the space of parameters for each fit) It

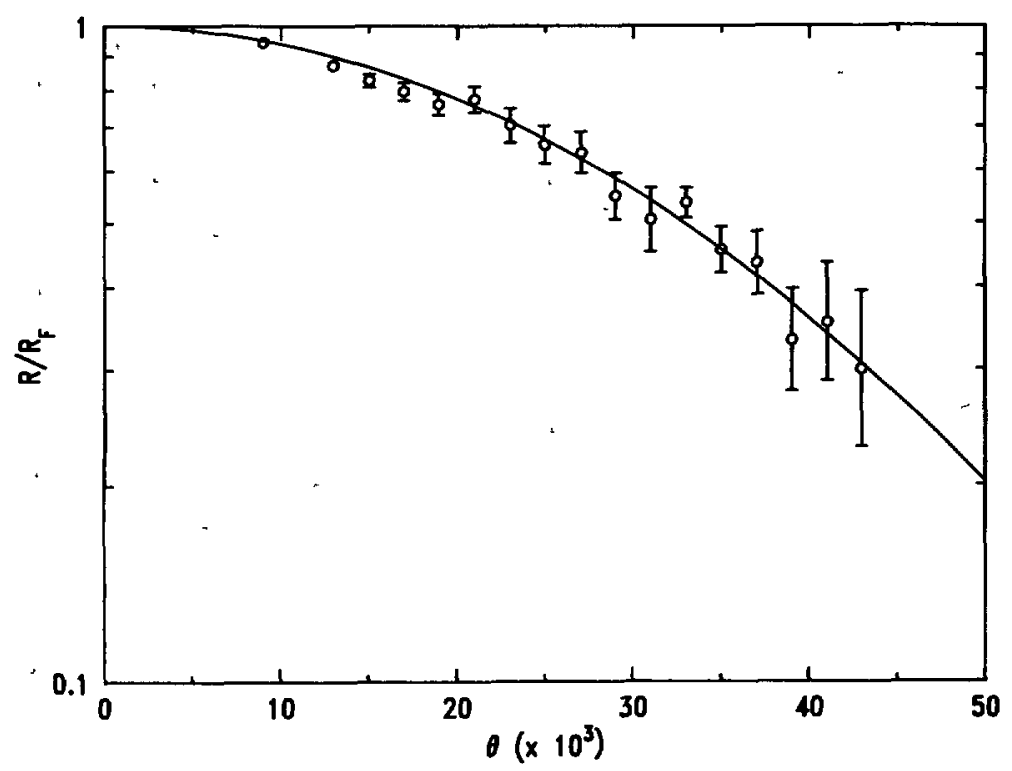

Fig 3 - Reflectivity curve of the bare water surface (normalized to Fresnel reflectivity) The line is a fit with,$\left\langle z^{2}\right\rangle^{1 / 2}=031 \mathrm{~nm}$ 
should be pointed out that this measured roughness is calculable from the coupling mode model for capillary waves without any adjustable parameter [39] indicating that this model provides a «good» cut-off for the modes at high wave-vectors (see the appendix).

\section{STRUCTURAL CHANGES.}

$31.1 \mathrm{LE} / \mathrm{LC}$ transition - The results concerning the LE/LC phase transition for hexadecanoic acid at $T=33{ }^{\circ} \mathrm{C}$ have been detailed elsewhere (Fig. 4, Tab. I) [25]. It occurs at a pressure below the spreading pressure and thus is an equilibrium phenomenon [36, 37] Let us recall that the transition is evidenced by an abrupt increase of the aliphatic medium thickness from $1.42 \pm 0.07 \mathrm{~nm}$ to $1.68 \pm 0.05 \mathrm{~nm}$ (Fig. 5), occurring àt constant density $\left(\rho_{\mathrm{c}}=(0.95 \pm 003) \rho_{\mathrm{H}_{2} \mathrm{O}}\right)$ Furthermore, the density $\rho_{\mathrm{h}}$ of the polar heads was found to be $1.24 \pm 0.03 \rho_{\mathrm{H}_{2} \mathrm{O}}$, and their thickness $\ell_{\mathrm{h}}$ slightly increased at the transition from $0.33 \pm 0.05 \mathrm{~nm}$ to $0.40 \pm 0.04 \mathrm{~nm}$, suggesting a reorientation. This, however, cannot be firmly established since $\rho_{\mathrm{h}}$ and $\ell_{\mathrm{h}}$ are correlated. Let us also note that the structural parameters of the film in the LC phase below the triple point (at $T=22{ }^{\circ} \mathrm{C}$ ) were found to be identical to those found above the triple point in the same phase, even at pressures for which the LE phase is present at $T=33{ }^{\circ} \mathrm{C}$ (Fig. 4).

We have also investigated the LE/LC transition of L- $\alpha$-DPPC, a compound which offers the possibility of an interesting companson with hexadecanoic acid, since the lengths of their aliphatic chains are identical, whereas their polar head-groups are quite different. This phospholipidic material allows the study of both the LE/LC and the LC/S transitions at the same temperature The data are reported in figure 4 and table II. In this case as well, the chain density remains constant over the LE and LC phases $\left(0.95 \rho_{\mathrm{H}_{2} \mathrm{O}}\right)$ : At the LE/LC transition, the aliphatic medium thickness increases from $15 \mathrm{~nm}$ to $1.7 \mathrm{~nm}$, and the density of the polar heads (which are much more important than in fatty acids) increases from $125 \rho_{\mathrm{H}_{2} \mathrm{O}}$ to $1.5 \rho_{\mathrm{H}_{2} \mathrm{O}}$

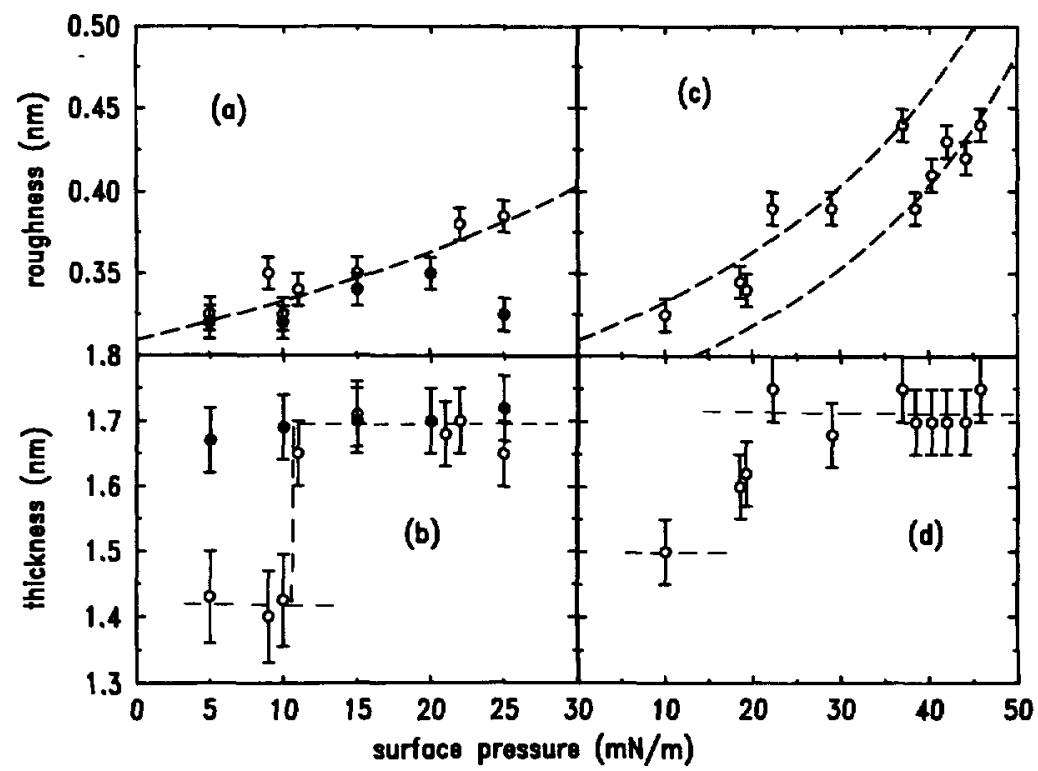

Fig 4 - Characteristics of the hexadecanoic acid film at $T=33{ }^{\circ} \mathrm{C}$ (hollow circles) and $T=22{ }^{\circ} \mathrm{C}$ (filled circles) (a) surface roughness, the dashed line is a fit using equation (3), (b) thickness of the alıphatic medium L- $\alpha$-DPPC $T=23{ }^{\circ} \mathrm{C}$ : (c) roughness, (d) thickness of the aliphatic medium 
Table Ia. - Parameters of the hexadecanoic actd monolayer at $T=33^{\circ} \mathrm{C}$

\begin{tabular}{|c|c|c|c|c|c|c|}
\hline$\Pi(\mathrm{mN} / \mathrm{m})$ & $\ell_{\text {tatls }}(\mathrm{nm})$ & $\delta_{\text {tatls }} \times 10^{6}$ & $\ell_{\text {heads }}$ & $\delta_{\text {heads }}$ & $\Gamma_{\text {heads }}\left(\mathrm{nm} \times 10^{6}\right)$ & $\left\langle 5^{2}\right\rangle^{1 / 2}(\mathrm{~nm})$ \\
\hline & $( \pm 01)$ & $( \pm 01)$ & $( \pm 01)$ & $( \pm 02)$ & $( \pm 02)$ & $( \pm 001)$ \\
5 & 143 & 34 & 033 & 44 & 1.45 & 0325 \\
9 & 1425 & 35 & 039 & 432 & 1.65 & 035 \\
10 & 1425 & 345 & 0325 & 44 & 145 & 0325 \\
11 & 165 & 345 & 033 & 44 & 145 & 033 \\
15 & 171 & 35 & 03 & 445 & 1.35 & 035 \\
21 & 1.7 & 33 & 045 & 445 & 2 & 035 \\
22 & 168 & 335 & 046 & 445 & 205 & 037 \\
25 & 169 & 33 & 039 & 43 & 17 & 038 \\
\hline
\end{tabular}

Table $\mathrm{Ib}$ - Parameters of the hexadecanoic acid monolayer at $T=22{ }^{\circ} \mathrm{C}$

\begin{tabular}{|c|c|c|c|c|c|c|}
\hline$\Pi(\mathrm{m} N / \mathrm{m})$ & $\ell_{\text {tatla }}(\mathrm{nm})$ & $\delta_{\text {tatla }} \times 10^{6}$ & $\ell_{\text {heads }}$ & $\delta_{\text {heads }}$ & $\Gamma_{\text {heads }}\left(\mathrm{nm} \times 10^{6}\right)$ & $\left\langle\mathrm{s}^{2}\right\rangle^{1 / 2}(\mathrm{~nm})$ \\
\hline & $( \pm 0.1)$ & $( \pm 01)$ & $( \pm 01)$ & $( \pm 02)$ & $( \pm 02)$ & $( \pm 001)$ \\
5 & 167 & 33 & 042 & 42 & 175 & 032 \\
10 & 169 & 35 & 043 & 44 & 190 & 032 \\
15 & 1705 & 345 & 033 & 44 & 145 & 0335 \\
20 & 169 & 34 & 038 & 435 & 165 & 0345 \\
25 & 172 & 345 & 041 & 44 & 180 & 032 \\
\hline
\end{tabular}

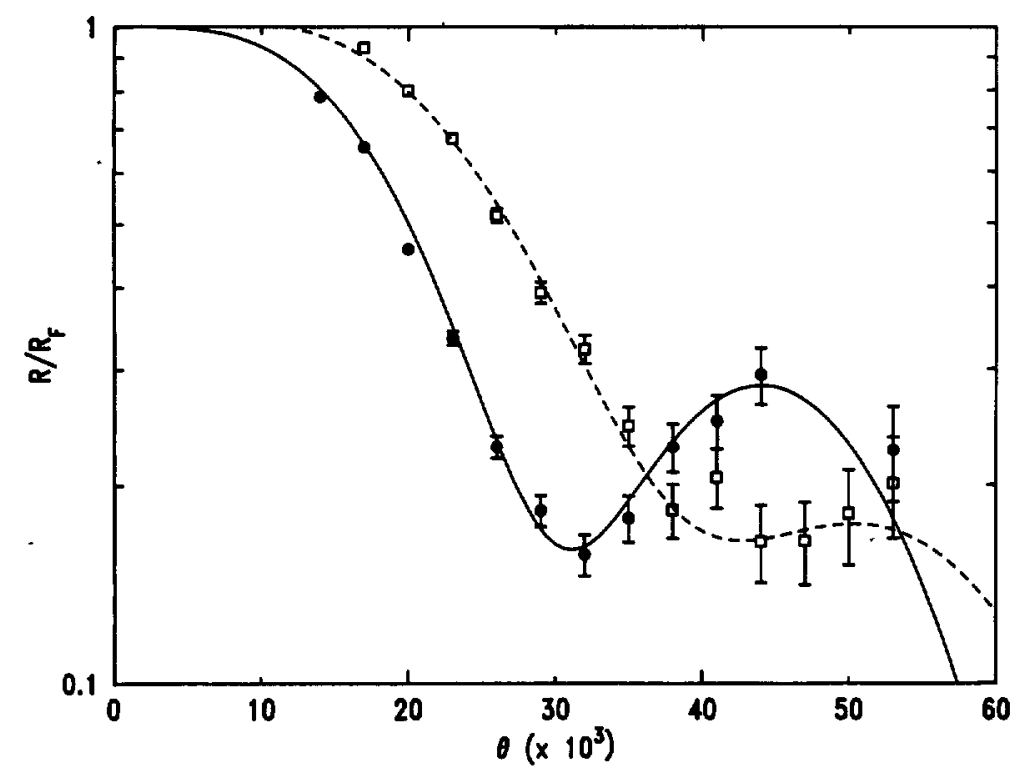

Fig 5 - Experimental reflectivity curves (normalized to Fresnel reflectıvity) for hexadecanoic acid and best fits. Hollow squares $T=33{ }^{\circ} \mathrm{C}, \quad \Pi=9 \mathrm{mN} / \mathrm{m}$ (LE phase) Filled circles $T=33{ }^{\circ} \mathrm{C}$, $\Pi=25 \mathrm{mN} / \mathrm{m}$ (LC phase) The shift in the destructive interference at $T=33{ }^{\circ} \mathrm{C}$ is indicative of a thicker LC film

The exact mechanism of the LE/LC transition remains a questionable subject [40]. It is generally admitted that in two dimensions rigid rods can only undergo one first-order phase transition, implying that at least one of the Gas/LE or LE/LC transition is to be attributed to 
Table II - Parameters of the $L-\alpha-D P P C$ monolayer at $T=23^{\circ} \mathrm{C}$

\begin{tabular}{|c|c|c|c|c|c|c|}
\hline$\Pi(m N / m)$ & $\ell_{\text {tarls }}(n m)$ & $\delta_{\text {tarls }} \times 10^{6}$ & $\ell_{\text {heads }}$ & $\delta_{\text {heads }}$ & $\Gamma_{\text {heads }}\left(n m \times 10^{6}\right)$ & $\left\langle\zeta^{2}\right\rangle^{1 / 2}(n m)$ \\
\hline & $( \pm 01)$ & $( \pm 01)$ & $( \pm 01)$ & $( \pm 04)$ & $( \pm 03)$ & $( \pm 001)$ \\
10 & 15 & 34 & 05 & 445 & 225 & 0325 \\
186 & 16 & 34 & 06 & 42 & 250 & 0345 \\
193 & 162 & 34 & 06 & 47 & 280 & 034 \\
22.3 & 1.75 & 34 & 06 & 54 & 325 & 039 \\
29 & 168 & 34 & 06 & 5 & 3 & 039 \\
37 & 175 & 34 & 06 & 535 & 320 & 044 \\
385 & 17 & 34 & 06 & 525 & 315 & 039 \\
403 & 17 & 34 & 06 & 55 & 330 & 041 \\
42 & 17 & 34 & 06 & 56 & 335 & 043 \\
441 & 17 & 34 & 06 & 55 & 330 & 04 \\
458 & 175 & 34 & 06 & 53 & 320 & 044 \\
\hline
\end{tabular}

the flexubility of the chains $[4,40,41]$. The present data suggest that such is the case for the LE/LC transition. Actually, if no conformational changes were to be involved, thermal motion of the chains as a whole would be responsible for the decrease in entropy at the transition [40], implying large changes in density, which are not observed. On the contrary, the increase in thickness exactly compensates for the reduction in available area under the constraint of constant density (Fig. 2). Let us note that the loss of conformational freedom can be balanced by an increase in dispersive-force strengths at nearly constant density since the film thickness increases.

The data also suggest a reorientation of the polar head-groups at the transition in the case of hexadecanoic acid Such a molecular reorientation has been observed at this transition by optical second-harmonic generation [18], and could arise from chain packing constraints On the contrary, in the case of DPPC (this determination is more accurate since the headgroups are more developed) no such reorientation is observed.

In the LC phase, the structure of the aliphatic medium appears to be independent on the temperature and on the nature of the polar headgroup, indicating that this phase should be characterized by a well defined conformation with only few defaults.

Recent molecular dynamics calculations [42] using a realistic model have furnished a picture very close to that suggested by the present results in the LE phase, each chain has one or two gauche conformations (decreasing the mean thickness), whereas in the LC phase, the molecules have less conformational defects and are tilted (here of approximately $30^{\circ}$ using a molecular length of $192 \mathrm{~nm}$ [43]). An identical conclusion has been drawn from a recent mean-field theory of chain packing statistics [40]

31.2 Liquid condensed and solid phase. - The thickness of the aliphatic medium and the roughness of the interface are reported in figure 6 and table III for behenic acid [24] The tail length increases slightly up to the solid phase with a more abrupt variation near the LC $\left(\mathrm{L}_{2}^{\prime}\right) / S$ transition Another increase $1 \mathrm{n}$ the thickness is observed at $\Pi=45 \mathrm{mN} / \mathrm{m}$, and $\mathrm{might}$ correspond to another phase transition, which has been inferred from symmetry arguments [7]. The phase at lower pressure should be-the "« superlıquid " LS phase in the nomenclature of Harkins [2, 5], and the other one the « solid» $S$ phase. As noticed in reference [21], the accuracy of the tail length determination is limited by coupling with the head group medium thickness, making the determination of very small tilt angles (up to $\approx 12{ }^{\circ}$ ) difficult. In any case, the tilt angle is very low in the solid S and LS phases Note also that the chain density remains almost constant, $\rho_{\mathrm{c}}=\left(\begin{array}{l}09 \pm 003\end{array}\right) \rho_{\mathrm{H}_{2} \mathrm{O}}$ over both the LC and $\mathrm{S}$ phases The density 


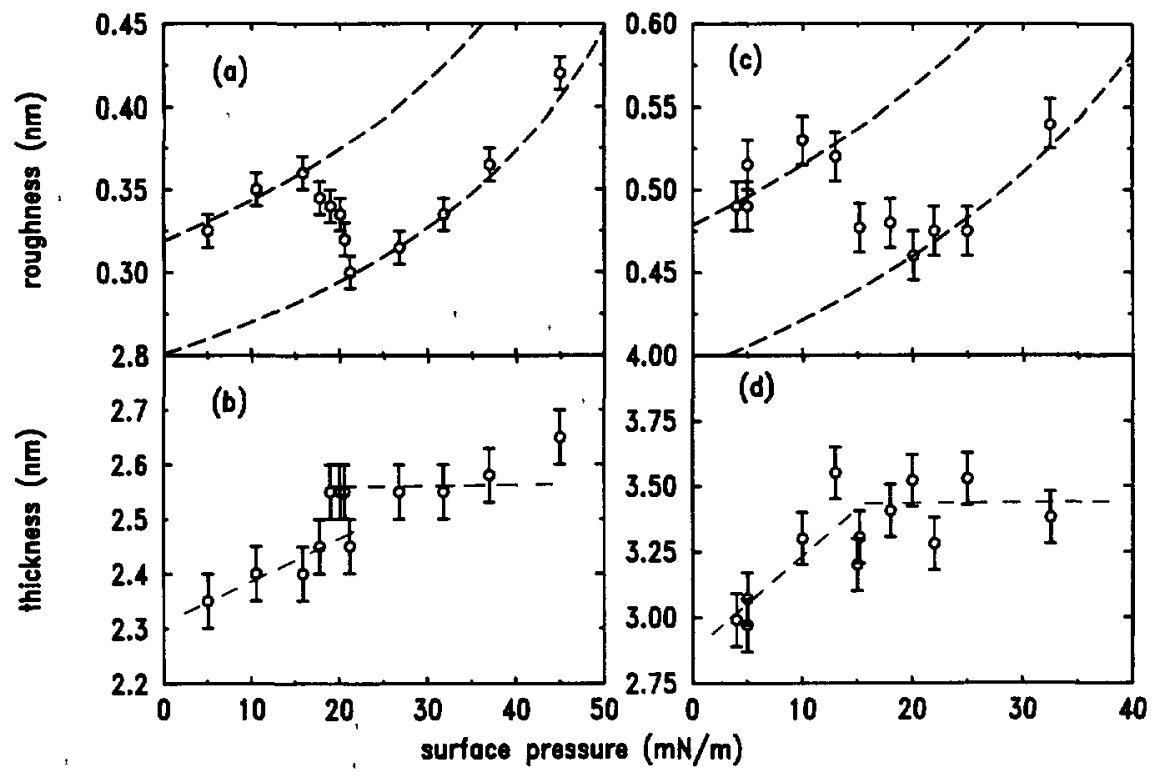

Fig 6 - Characteristics of the behenic acid film at $T=23{ }^{\circ} \mathrm{C}$ (a) surface roughness, the dashed line is a fit usıng equation (3), (b) thickness of the aliphatic medium. Melissic acid $T=23{ }^{\circ} \mathrm{C}$ (c) roughness, (d) thıckness of the aliphatic medium

Table III. - Parameters of the behenic actd monolayer at $T=23{ }^{\circ} \mathrm{C}$

\begin{tabular}{|c|c|c|c|c|c|c|}
\hline$\Pi(\mathrm{mN} / \mathrm{m})$ & $\ell_{\text {tall } s}(\mathrm{~nm})$ & $\delta_{\text {tauls }} \times 10^{6}$ & $\ell_{\text {heads }}$ & $\delta_{\text {heads }}$ & $\Gamma_{\text {heads }}\left(\mathrm{nm} \times 10^{6}\right)$ & $\left\langle\mathrm{s}^{2}\right\rangle^{1 / 2}(\mathrm{~nm})$ \\
\hline & $( \pm 015)$ & $( \pm 01)$ & $( \pm 006)$ & $( \pm 03)$ & $( \pm 02)$ & $( \pm 007)$ \\
5.1 & 235 & 35 & 025 & 5 & 125 & 0325 \\
106 & 24 & 36 & 025 & 5 & 125 & 035 \\
159 & 24 & 34 & 025 & 51 & 130 & 036 \\
178 & 245 & 35 & 03 & 51 & 150 & 0345 \\
19 & 255 & 35 & 025 & 51 & 130 & 034 \\
201 & 255 & 35 & 025 & 53 & 135 & 0335 \\
206 & 255 & 355 & 025 & 53 & 135 & 032 \\
212 & 245 & 3.4 & 04 & 52 & 210 & 03 \\
268 & 255 & 325 & 03 & 53 & 160 & 0315 \\
318 & 245 & 33 & 04 & 52 & 210 & 0335 \\
37 & 258 & 355 & 04 & 53 & 210 & 0365 \\
45 & 265 & 36 & 033 & 57 & 190 & 042 \\
\hline
\end{tabular}

of the polar heads also remains approximately constant $\rho_{\mathrm{h}} \approx 1.5 \rho_{\mathrm{H}_{2} \mathrm{O}}$, whereas their thıckness increases from $025 \mathrm{~nm}$ to $0.4 \mathrm{~nm}$, suggesting a reorientation

The evolution on compression of the melissic acid film is similar to that of behenic acid (Fig. 6, Tab IV). The chain length increases rather smoothly near the transition from $\approx 3 \pm 002 \mathrm{~nm}$ to $\approx 35 \pm 002 \mathrm{~nm}$. The chain density slightly increases from $\rho_{\mathrm{c}}=(092 \pm 002) \rho_{\mathrm{H}_{2} \mathrm{O}}$ in the LC phase and remains constant within the solid phase $\rho_{\mathrm{c}}=(0.97 \pm 002) \rho_{\mathrm{H}_{2} \mathrm{O}}$, whereas the density of the $038 \pm 0.04 \mathrm{~nm}$ thick heads increases contınuously from $118 \rho_{\mathrm{H}_{2} \mathrm{O}}$ to $1.47 \rho_{\mathrm{H}_{2} \mathrm{O}}$ 
Table IV - Parameters of the triacontanoic actd monolayer at $T=23{ }^{\circ} \mathrm{C}$

\begin{tabular}{|c|c|c|c|c|c|c|}
\hline$\Pi(\mathrm{mN} / \mathrm{m})$ & $\ell_{\text {tasis }}(\mathrm{nm})$ & $\delta_{\text {tavls }} \times 10^{6}$ & $\ell_{\text {heads }}$ & $\delta_{\text {heads }}$ & $\Gamma_{\text {head }}\left(\mathrm{nm} \times 10^{6}\right)$ & $\left\langle\varsigma^{2}\right\rangle^{1 / 2}(\mathrm{~nm})$ \\
\hline & $( \pm 01)$ & $( \pm 01)$ & $( \pm 006)$ & $( \pm 03)$ & $( \pm 02)$ & $( \pm 0015)$ \\
4 & 299 & 333 & 039 & 447 & 175 & 048 \\
5 & $297-$ & 3.27 & 044 & 427 & 190 & 049 \\
5 & 307 & 346 & 0392 & 412 & 160 & 0515 \\
10 & 33 & 332 & 038 & 47 & 180 & 053 \\
13 & 355 & 353 & 039 & 485 & 190 & 052 \\
15 & 33 & 34 & 038 & 445 & 170 & 0475 \\
15 & 337 & 345 & 037 & 46 & 170 & 048 \\
18 & 345 & 345 & 038 & 473 & 180 & 048 \\
20 & 351 & 351 & 041 & 48 & 200 & 046 \\
22 & 328 & 3.32 & 04 & 53 & 200 & 047 \\
25 & 352 & 34 & 036 & 48 & 175 & 0475 \\
325 & 338 & 327 & 035 & 48 & 170 & 0535 \\
\hline
\end{tabular}

In both cases, the continuous variations of the film parameters reflect the decrease in molecular area It appears that the constraint $\mathcal{A}=n_{\mathrm{c}} / \rho_{\mathrm{c}} \ell_{\mathrm{c}}$ is mainly filled by an increase in the film thickness. The results support a reorientation of both the aliphatic chains and polar headgroups within the LC phase

313 Multulayering $t$ melissic aczd - The reflectivity curves reported in figure 7 have been recorded at $\Pi=20 \mathrm{mN} / \mathrm{m}$ and $37.5 \mathrm{mN} / \mathrm{m}$, in the solid phase The curve recorded at $\Pi=20 \mathrm{mN} / \mathrm{m}$ can easily be fitted using equation (2), with $\rho_{\mathrm{c}}=098 \rho_{\mathrm{H}_{2} \mathrm{O}}, \ell_{\mathrm{c}}=35 \mathrm{~nm}$, $\rho_{\mathrm{h}}=137 \rho_{\mathrm{H}_{2} \mathrm{O}}, \quad \ell_{\mathrm{h}}=038 \mathrm{~nm}$ and $\sigma=0.47 \mathrm{~nm}$. Obviously, the curve recorded at $\Pi=375 \mathrm{mN} / \mathrm{m}$ cannot be fitted with such a simple model. The peak at 24 mrad is much sharper, and its shape is different (see details near $15 \mathrm{mrad}$ and $30 \mathrm{mrad}$ ). Actually, one can give account of these features assuming that a part of the surface is covered with more than a monolayer. Interference patterns are given in figure $7 \mathrm{c}$ for 3, 4 and 21 layers (representing

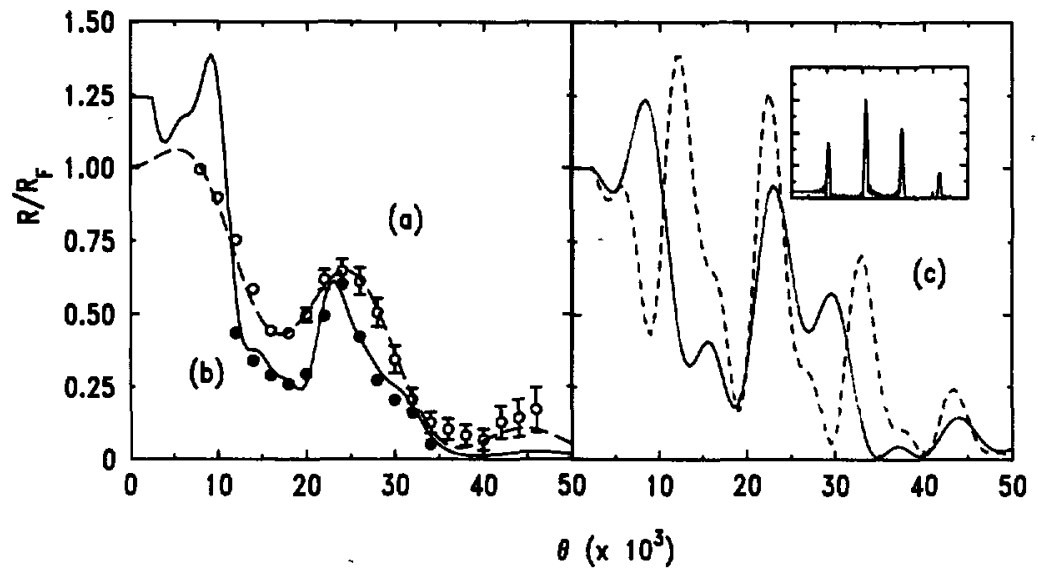

Fig. 7. - Experimental reflectivity curves (normalized to Fresnel reflectivity) for the melissic acid film The curves were recorded at (a) $\Pi=20 \mathrm{mN} / \mathrm{m}$, (b) $\Pi=375 \mathrm{mN} / \mathrm{m}$, the dashed line is a fit, and the solıd line has been calculated following the ideas of $\&$ III 3 (c) calculated reflectivity curves for multılayers of melissic acid on water full line 3 layers; dashed line : 4 layers, insert 21 layers 
crystallites) Clearly, an even number of layers (1 e. with polar groups on the top of the stackıng) would cause a resulting shape of the reflectivity curve that would be inconsistent with the experimental data. Moreover, since the particular shape obtained for an odd number of layers is due to the presence of only one additional layer, it tends to a classical (1.e. also inconsistent with the data) Bragg pattern for an increasing number of layers This indicates that the monolayer is in equilibrium with mult1layers composed of a small (mainly 3 or 5) odd number of layers. It is generally assumed, but, to our knowledge, without direct experimental basis, that, such structures are involved in the "collapse " of the monolayer According to the non-direct determination of reference [6], an even number of layers is allowed in the case of fatty esters, probably because of a lower surface energy This transition to a multilayer can be related to the plateaux which are apparent on a few $(I, \mathcal{A})$ curves, at pressures well below the limit of stability of the film; such layers exhibiting nucleation were rejected for X-ray experıments. However, above their spreadıng pressure, monolayers are metastable (their metastability depends whether or not the time of observation is larger than the characteristic time for molecular relaxation, which decreases with increasing pressure [44]) and recent careful experiments [44] have shown hat nucleation would probably occur in our case.

32 BENDING RIGIDITY OF MONOLAYERS IN THE SOLID PHASE. - If the roughness of the film has been measured for a given resolution of the diffractometer, and if the surface pressure is determined otherwise (Wilhelmy balance), $K$ can be calculated from equation (3). Since a drop in the roughness larger than $0.02 \mathrm{~nm}$ can be detected, rigidities larger than $k_{\mathrm{B}} T$ can be determined. This method is indeed sensitive to the bending rigidity modulus since the cut-off at high wave-vectors due to rigidity $\sqrt{\gamma / K} \approx 3 \times 10^{9} \mathrm{~m}^{-1}$ is smaller than the molecular cut-off $2 \pi$ /a (a is a molecular dimension), see the appendix. Since $K$ appears in equation (3) with a logarithmic dependence, the precision of its determination is generally low. By derivation of equation (3) one gets $\Delta K / K \approx\left(8 \pi \gamma / k_{\mathrm{B}} T\right) \sigma \Delta \sigma$, if the incertitude on $\sigma$ is as low as $0.01 \mathrm{~nm}$, one should nevertheless have $\Delta K / K \approx 05$

Behentc actd. In the LC phase, the roughness increases upon compression as $1 / \sqrt{\gamma}$, extrapolating to the roughness of water at $\Pi=0$ (Fig 6a) More precisely, the measured roughness follows equation (3), and thus the sole contribution of capillary waves without additional rigidity accounts for it. According to reference [45] the roughness follows $\Pi$ More recently published data by the same group are, however, in full agreement with a $1 / \sqrt{\gamma}$ in the LC phase [21] The striking drop in the roughness at the LC/S transition (Fig. 8) $(\Pi=20 \mathrm{mN} / \mathrm{m})$ is due to the appearence of a high bending rigidity in the solid phase [24], which can be estimated using equation (3) $K=170 k_{\mathrm{B}} T$, the experimental data are consistent with a rather wide range of bending nigidity values, between $120 k_{\mathrm{B}} T$ and $300 k_{\mathrm{B}} T$ After the transition, the roughness again increases as $1 / \sqrt{\gamma}$, and thus $K$ remains constant within experimental incertitude

15 alkyl-group compounds The variations of the roughness of the L- $\alpha$-DPPC film (Fig. 4c) are similar to those obtained with behenic acid The drop in the roughness at $\Pi=35 \mathrm{mN} / \mathrm{m}$ (LC/S transition) corresponds to the appearence of a bending nigidity $K=15 k_{\mathrm{B}} T$ (with $\left.4 k_{\mathrm{B}} T \leqslant K \leqslant 70 k_{\mathrm{B}} T\right)$ Both below and above the triple point, $1 \mathrm{e}$ in the LE and LC phases, the roughness of the hexadecanoic acid film increases as $1 / \sqrt{\gamma}$, extrapolating to the roughness of water At $T=22^{\circ} \mathrm{C}$, a significantly lower roughness was obtained at the limit of stability of the layer $(\Pi=25 \mathrm{mN} / \mathrm{m})$, which, using equation (3), corresponds to a bending rigidity $K=70 k_{\mathrm{B}} T$

Melissic actd The variations of the roughness (Fig $6 \mathrm{c}$ ) are also similar to those observed with behenic acid. Their values, however, which are approximately $0.15 \mathrm{~nm}$ larger for similar surface tensions, imply a stronger scattering by surface irregularities, and thus another 


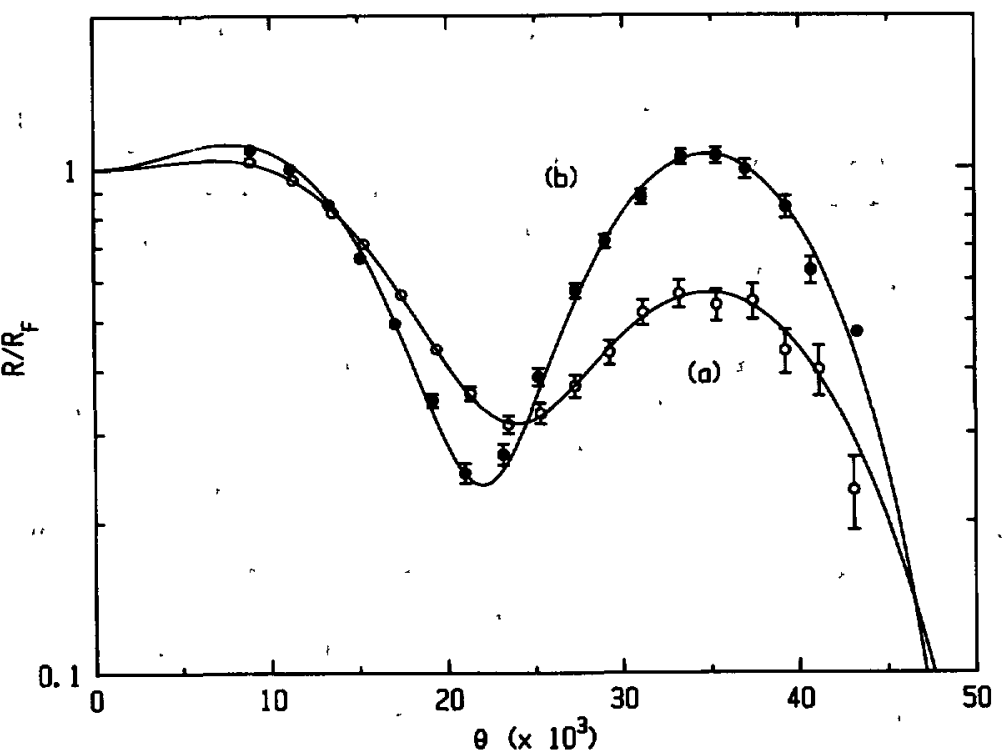

Fig 8 - Measured reflectivity of the monolayer of behenic acid (a) in the LC phase $(\Pi=15 \mathrm{mN} / \mathrm{m})$, (b) in the solıd phase $(I I=22 \mathrm{mN} / \mathrm{m})$, divided by the Fresnel reflectıvity of water $R_{\mathrm{F}}$, and the best fits $\left\langle z^{2}\right\rangle^{1 / 2}=0355 \mathrm{~nm}$ at $15 \mathrm{mN} / \mathrm{m}$ and $0.3 \mathrm{~nm}$ at $22 \mathrm{mN} / \mathrm{m}$.

contribution to be added to that of capillary waves From the preceding, one can expect that this contribution comes, at least partly, from small multılayer domains. Another possibility is that of an intrinsic roughness of the monolayer [40], which would be' overwhelmed by capillary waves for shorter chains. Experıments on transferred films are to be undertaken in order to clarify this important point related to chain statistics Assuming $\mathrm{a} \approx 0.12 k_{\mathrm{B}} T$ (appendix) bending rigidity in the LC phase, as in the case of behenic and hexadecanoic acid, one can estimate (using $\sigma_{\text {measured }}^{2}=\sigma_{\text {capillary }}^{2}+\sigma_{\text {add }}^{2}$ [32] this additional roughness to be $\sigma_{\text {add }}=038 \pm 0.03 \mathrm{~nm}$ Since this value is approximately constant in the LC phase, and since no dramatic structural changes are observed at the LC/S transition, one can expect that this estimation is also relevant for not too high surface pressures in the solid phase. One can then get a crude estimation of $K, 300 k_{\mathrm{B}} T \leqslant K \leqslant 1000 k_{\mathrm{B}} T$

'Comparison with bending rigldity values in similar systems.

$X$-ray reflectıvity is, to our knowledge, unique in allowing the determination of high bending rigidities of the magnitude of those of insoluble monolayers. In light scattering experiments, the observation of capillary waves is limited to wavelengths of approximately $1 \mu \mathrm{m}$, a value which is much larger than $\sqrt{K / \gamma}$. Ellipsometric measurements are very sensitive to the large fluctuations of interfaces of very low surface tension and bending rigidity, since, at Brewster angle, the contribution of capillary waves to the ellipticity (which is imaginary)' is directly proportional to $K^{-1 / 2}(\bar{\rho} \propto 1 / \sqrt{K \gamma})[46,47]$. For capillary waves at the air/water interface with $\lambda=500 \mathrm{~nm}$, however, this contribution is of the order of the experimental incertitude $[46 ; 48]$

Mult1-membrane systems with low surface tension have been extensively studied by various methods, and the values obtained with these systems provide interesting comparisons to our data Brochard et al. [49] have shown by analyzing the flicker intensity of red blood cells that $K \approx 5$ to $20 k_{\mathrm{B}} T$ for this system. $K$ has also been determined by direct [50] or Fourier [51] analysis of the thermal undulations of vesicles or other methods such as the micropipette 
technique [52]. The values obtained using all these techniques range between 5 : and $20 k_{\mathrm{B}} T$ for molecules having between 10 and 20 monomers. Bending rigidity values have also been measured in multimembranes lamellar phases using ESR [53], X-ray diffraction [54] or light scattering [55] (since here $\gamma$ is very low, $\sqrt{K / \gamma}$ is much larger than for monolayers) A cosurfactant is generally used to stabilize such lamellar systems, leading to a bending rigidity $\approx k_{\mathrm{B}} T$ approximately one order of magnitude lower than for pure surfactant [54].

Discussion.

The questions to be adressed in this last paragraph are Why does the film in the solid phase exhibit a large bending rigidity modulus? Is it possible to understand its value and variations with chain length? This problem has recently been tackled for bilayer membranes [56] and polymer brushes [57], but it is not clear whether or not these approaches apply to the case of monolayers. In any case, these theories give a thickness dependence similar to that of the theory of elasticity for continuous media. The free energy of a plate bent along $x$ is [58]

$$
F=\frac{E h^{3}}{24\left(1-\sigma_{\mathrm{P}}^{2}\right)} \int \mathrm{d} x\left(\frac{\partial^{2} \zeta}{\partial x^{2}}\right)^{2}
$$

where $\zeta(x)$ is the deformation of the plate of thickness $h, E$ the Young modulus, and $\sigma_{\mathrm{P}}$ the Poisson modulus. This dependence comes from the plate being stretched at some points and compressed at others Defining the bending rigidity $K$ by $F=1 / 2 K C^{2}$ where $C$ is the mean curvature of the surface, one obtains

$$
K=\frac{E h^{3}}{12\left(1-\sigma_{\mathrm{P}}^{2}\right)}
$$

$E /\left(1-\sigma_{\mathrm{P}}^{2}\right)$ can be calculated from the dilational modulus of the film $\varepsilon=-\dot{A}(\partial \Pi / \partial A)_{T}[15$, 59] $\varepsilon \approx 1.1 \mathrm{Nm}^{-1}$ for stearic acid [2], and thus $E /\left(1-\sigma_{\mathrm{P}}^{2}\right)=\varepsilon h^{-1} \approx 5.10^{8} \mathrm{~J}_{\mathrm{m}} \mathrm{m}^{-3}$. Using (5),

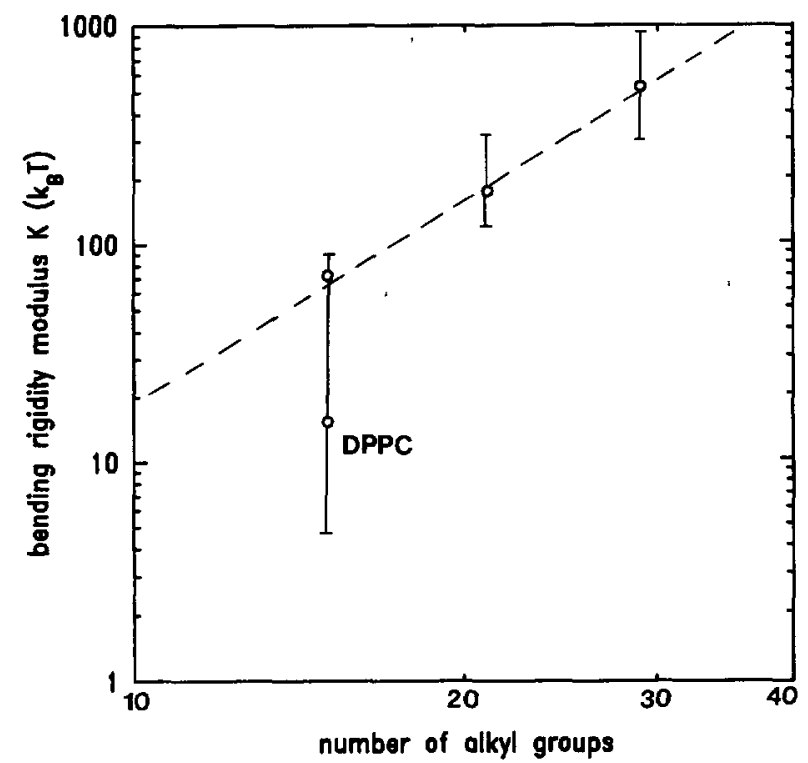

Fig 9 - Bending rigidity modulus as a function of the number of alkyl groups The points are the experimental results for $\log K$ and the full line has been calculated using equation (5) with no adjustable parameter 
one finds $K=190 k_{\mathrm{B}} T$ for behenic acid. Moreover, the $h^{3}$ dependence is consistent with the experimental data (Fig. 9) The appearance of a high bending rigidity in the solid phase is thus explained by the very low compressibility of this phase. It should also be noticed that a realistic value for the prefactor in equation (5) can be obtained from the microscopic model of reference [57], retaining only an entropic contribution [60]. Although this model considers thickness variations at constant density, it is equivalent to lowest order to that developed in reference [58].

\section{Concluding remarks}

Among the results presented above, the constancy of the aliphatic medium density within the liquid and solid phases appears to be a most general feature of organic films adsorbed on water From this constraint follows the abrupt increase in thickness at the LE/LC transition and the continuous one within the LC phase The transition to the solid phase is characterized at a microscopic level by a more subtle change, the damping of capillary waves, implying the appearance of a high bending rigidity, which is related to the elastic properties of the monolayer.

\section{Acknowledgements.}

We wish to thank A. Braslau for a very critıcal reading of the manuscript. and O Bélorgey for fruitful discussions

\section{Appendix.}

\section{A1. Statistical mechanics of capillary waves.}

The purpose of this appendix is to calculate the roughness due to thermally excited capilary waves in the case of a rigid interface and to estimate the value to be obtained from X-ray experiments, taking into account the resolution of the diffractometer

An analysis of the intensity scattered by the surface gives information on the power spectrum $|\tilde{z}|^{2}$ (modes $\kappa_{x}$ and $\kappa_{y}$ ) of the roughness :

$$
\tilde{z}\left(\kappa_{x}, \kappa_{y}\right)=1 / S \iint_{S} \mathrm{~d} x \mathrm{~d} y z(x, y) \mathrm{e}^{-2 \imath \pi\left(\kappa_{x} x+\kappa_{y} y\right)}
$$

where $z(x, y)$ is the height of the interface at $(x, y)$.

The most important characteristics of the height distribution $z(x, y)$ are its standard deviation $\left\langle z^{2}\right\rangle^{1 / 2}$ and the normalized autocorrelation function $\mathcal{C}(\tau)$ (owing to the cylindrical symmetry, $\mathfrak{C}$ only depends on $\tau=\sqrt{x^{2}+y^{2}}$ )

$$
\mathcal{E}(\tau)=\frac{\langle z(0) \cdot z(\tau)\rangle}{\left\langle z^{2}\right\rangle}=\frac{\iint \mathrm{d} \kappa_{x} \mathrm{~d} \kappa_{y} J_{0}\left(\tau \sqrt{\kappa_{x}^{2}+\kappa_{y}^{2}}\right)\left|\tilde{z}\left(\kappa_{x}, \kappa_{y}\right)\right|^{2}}{\iint \mathrm{d} \kappa_{x} \mathrm{~d} \kappa_{y}\left|\tilde{z}\left(\kappa_{x} \kappa_{y}\right)\right|^{2}}
$$

where $J_{0}$ s the Bessel function of the first kind of order 0 ; the power spectrum of capillary waves $\left|\tilde{z}\left(\kappa_{x}, \kappa_{y}\right)\right|^{2}$ is [39]

$$
\left|\tilde{z}\left(\kappa_{x}, \kappa_{y}\right)\right|^{2}=\frac{k_{\mathrm{B}} T}{4 \pi \gamma} \frac{1}{\Delta \rho g+\gamma \kappa^{2}+K \kappa^{4}}
$$


$\gamma$ is the surface tension and $K$ the bending rigidity of the interface Note that, due to a coupling between modes [39], every interface exhibits at least a small bending rigidity, which according to a simple calculation, is equal to $(3 / 8 \pi) k_{\mathrm{B}} T$

A conclusive experimental proof of equation (A 3) can only be provided by carefully examining the intensity scattered outside the specular direction, and we hope to successfully tackle this problem in future experiments. In any case, the surface tension $\left(\kappa^{2}\right)$ term is dominant at higher compressibilities (liquid phases), and this is demonstrated by the measured $1 / \sqrt{\gamma}$ increase of the roughness. Otherwise, the free energy contains rigidity $\left(\kappa^{4}\right)$ terms which have been kept in our analysis, and eventually $\kappa^{3}$ as well as higher power terms ; such terms may anse from the electric properties of membranes [61, 62]. Flexoelectricity (polarization induced by to curvature) renormalizes the bending rigidity $K_{\text {eff }}=K+e_{\mathrm{f}}^{2} \Lambda / \varepsilon_{0} \varepsilon_{\mathrm{r}}$, where $e_{\mathrm{f}}$ is the flexoelectric coefficient on the order of $1.5 \times 10^{-20} \mathrm{C}$ [63] and $A^{-1}$ is of the order of the layer thickness $(\approx 25 \mathrm{~nm})$. Therefore $4 \pi e^{2} \Lambda / \varepsilon_{0} \varepsilon_{r} \approx 003 k_{\mathrm{B}} T$ is negligible in our case The $\kappa^{5}$ term arising from polarization charge-polarization charge interactions [61] is of the order of $10^{-12} \kappa$ and may be neglected. The effect of permanent dipoles depends on their orientation with respect to the surface of water. For dipoles parallel to the surface, height and polarisation fluctuations are not coupled [61]. For dipoles normal to the surface, a $\kappa^{3}$ term appears, which is obviously dominated by the surface tension $\left(\kappa^{2}\right)$ term over the range of wave-vectors investigated The ratio of the $\kappa^{3}$ term to the $\kappa^{4}$ term is $p_{0}^{2} / 2 \varepsilon_{0} \varepsilon_{\mathrm{r}} K \kappa$ With $p_{0} \leqslant 1 \mathrm{D} / \mathrm{nm}^{2}\left(p_{0} \approx 0.1 \mathrm{D} / \mathrm{nm}^{2}\right.$ for behenic acid [64]), the $\kappa^{4}$ term dominates for wave-vectors larger than $\approx 10^{5} \mathrm{~m}^{-1}$ (corresponding to our resolution) and for the high bending rigidities that we examıne. In any case, the experimental results with a drop in the roughness correlated to the decrease in compressibility at the transition (together with the $1 / \sqrt{\gamma}$ increase of the roughness in liquid phases) strongly supports our treatment which consists in only retainıng elastic terms

Using (A 2) and (A 3) one can compute the roughness of the interface due to capillary waves (the standard deviation of the distribution)

$$
\sqrt{\left\langle z^{2}\right\rangle}=\left(\frac{k_{\mathrm{B}} T}{4 \pi \gamma} \log \sqrt{\frac{\gamma^{2}}{\Delta \rho g K}}\right)^{1 / 2}
$$

For water, with $\gamma=72 \mathrm{mN} / \mathrm{m}, K=3 / 8 \pi k_{\mathrm{B}} T, \sqrt{\left\langle z^{2}\right\rangle}=0392 \mathrm{~nm}$ at $T=25^{\circ} \mathrm{C} \quad$ A straightforward calculation also gives .

$$
\mathcal{C}(\tau)=\frac{K_{0}(\tau \sqrt{\Delta \rho g / \gamma})-K_{0}(\tau \sqrt{\gamma / K})}{\log \left(\sqrt{\gamma^{2} / \Delta \rho g K}\right)}
$$

where $K_{0}$ is the modified Bessel function of the second kind of order $0 \mathrm{C}(\tau)$ is represented in figure $10\left(\sqrt{\gamma / K)^{-1}}\right.$ is the cut-off at short distances (high wave-vectors) due to rigidity, and $(\sqrt{\Delta \rho g / \gamma})^{-1}$ the gravitational cut-off at low wave-vectors Since $K_{0}(z) \sim-\log z$ and $\lim K_{0}(z)=0, \mathcal{C}(\tau)$ can be approxımated as follows . $z \rightarrow \infty$

$$
\mathcal{C}(\tau) \approx\left\{\begin{array}{cc}
1 & \tau<\sqrt{K / \gamma} \\
1-\frac{\log (\tau \sqrt{\gamma / K})}{\log \left(\sqrt{\gamma^{2} / \Delta \rho g K}\right)} & \sqrt{K / \gamma}<\tau \sqrt{\gamma / \Delta \rho g} \\
0 & \tau>\sqrt{\gamma / \Delta \rho g}
\end{array}\right.
$$

Note that this function is not divergent at $\tau \rightarrow 0$. 


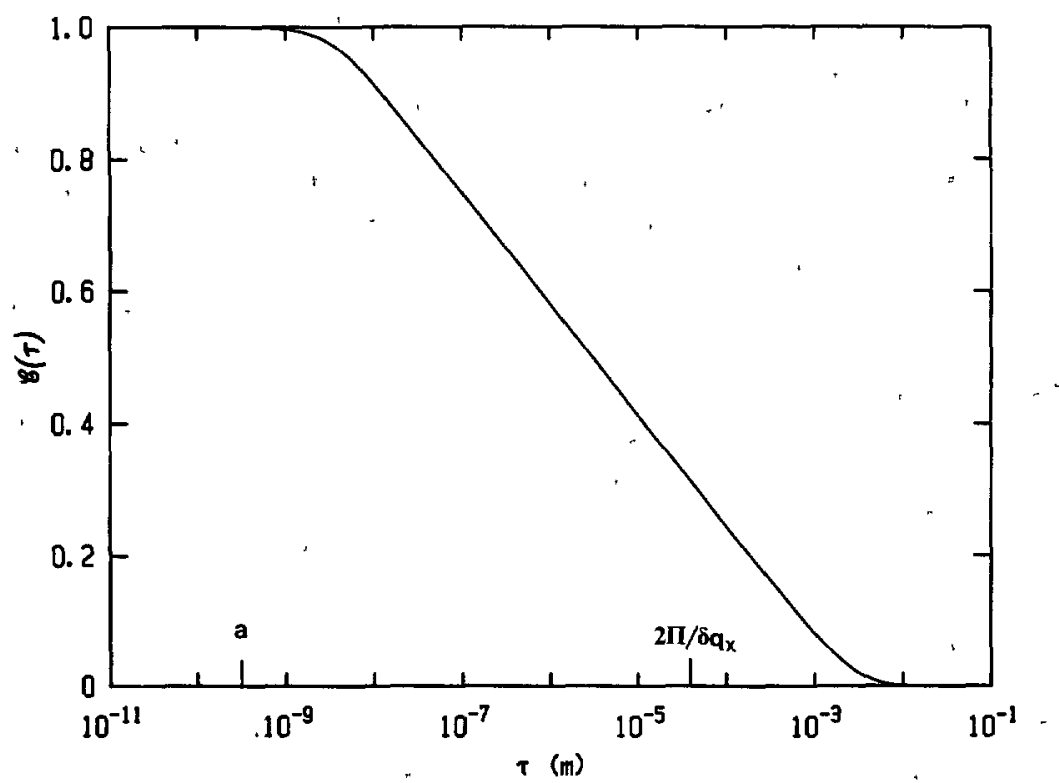

Fig 10 - Normalized autocorrelation function calculated for capillary waves from equation (A 5) a is the distance between alıphatıc chains in a condensed layer

\section{A2. Scattering by surface irregularities.}

Using the Kirchhoff theory [65] or other methods [32, 66, 67] (all these methods are equivalent after the required approximations are performed and amount to computing the phase differences, due to the roughness, of plane waves reflecting on a perfect diopter), one obtains for the intensity scattered in the direction $q^{\text {. }}$

$$
I_{\text {scatt }} \propto \iint \mathrm{d} x \mathrm{~d} y \mathrm{e}^{l\left(q_{x} x+q_{y} y\right)}\left\langle\mathrm{e}^{l q_{z}[z(x, y)-z(0,0)]}\right\rangle
$$

Assuming a normal distribution for $z(x, y)$ (this is rigorous for an interface described-by a harmonical Hamiltonian, here in the limit $\tau \geqslant \sqrt{K / \gamma}$, the region that accounts for almost all the scattered intensity), the mean value of the phase difference in equation (A 7 ) is given by

$$
\left\langle\mathrm{e}^{l q_{z}[z(x, y)-z(0,0)]}\right\rangle=\mathrm{e}^{-q_{z}^{2}\left\langle z^{2}\right\rangle(1-e(x, y))}
$$

and thus,

$$
I_{\text {scatt }} \propto \int^{\cdot} \tau \mathrm{d} \tau J_{0}\left(\tau \sqrt{q_{x}^{2}+q_{y}^{2}}\right) \mathrm{e}^{-q_{z}^{2}\left\langle z^{2}\right\rangle(1-e(\tau))}
$$

(A 9) shows that for given $q_{x}$ and $q_{y}$, the contribution due to surface irregularities will only be significative if these irregularities are separated by less than $\left(q_{x}^{2}+q_{y}^{2}\right)^{-1 / 2}$ (and a high value of the correlation function)

In the $q_{z}$ direction $\left(\dot{q}_{x}=0, q_{y}=0\right)$ the information about the roughness spectrum is lost (this is an obvious property of the Fourier transform). The morphology $z(x, y)$ cannot be studied by such a reflectivity experiment, and one describes the interface using a density distribution $\rho(z)$ This justifies the method described in section I to compute the reflectivity 
For a Gaussian height distribution, $\rho(z)$ is an error function whose width is the measured roughness [32].

\section{A3. Influence of the diffractometer resolution on the measured roughness.}

In fact, the angular resolution of the diffractometer is finite, and, thus, (A 7) has to be summed over a range of $q$. Near the specular condition, the proportionality factor in (A 7) reduces to $R_{\mathrm{F}}(\theta)$, and then, the reflectivity is the convolution .

$$
R\left(q_{z}\right)=R_{\mathrm{F}}\left(q_{z}\right) \iint \mathrm{d} q_{x} \mathrm{~d} q_{y} \iint \mathrm{d} x \mathrm{~d} y \mathrm{e}^{l\left(q_{x} x+q_{y} y\right)} \mathrm{e}^{-\left\langle z^{2}\right\rangle(1-\mathrm{e}(x, y)) q_{z}^{2}} \mathcal{R}\left(q_{x}, q_{y}\right)
$$

where $\mathcal{R}$ is the resolution function of the diffractometer, for which we assume a Gaussian shape [32]. Let us note that (A 10) only holds when the dimensions of the llluminated area are much smaller than the source-to-sample and sample-to-detector distances, and that this is not verified at too grazıng angles of incidence (lower than $\approx 10^{-2}$ rad) Performing the integration over $q_{x}$ and $q_{y}$.

$$
R\left(q_{z}\right)=R_{\mathrm{F}}\left(q_{z}\right) \iint \mathrm{d} x \mathrm{~d} y \mathrm{e}^{-q_{z}^{2}\left\langle z^{2}\right\rangle(1-\mathrm{e}(x, y))} \tilde{R}(x, y)
$$

where the Fourier transform of $\mathcal{R}$ is $\tilde{\mathcal{R}}$ :

$$
\tilde{\mathcal{R}}(x, y)=\frac{\Delta q_{x} \Delta q_{y}}{2 \pi} \mathrm{e}^{-1 / 2\left(\Delta q_{x}^{2} x^{2}+\Delta q_{y}^{2} y^{2}\right)}
$$

where $\Delta q_{x} / \sqrt{2 \log 2}$ and $\Delta q_{y} / \sqrt{2 \log 2}$ are the half-widths at half-maximum of the resolution function $\mathcal{R}$ along the $x$ and $y$ axis. Inserting (A 6) in (A 11), taking into account that $\Delta q_{x} \Delta q_{y} \gg \gamma / \Delta \rho g$, it follows

$$
\begin{aligned}
& R\left(q_{z}^{\gamma}\right)=R_{\mathrm{F}}\left(q_{z}\right)\left\{\int_{-\sqrt{K / \gamma}}^{\sqrt{K / \gamma}} \mathrm{d} x \mathrm{~d} y \tilde{\mathcal{R}}(x, y)+\cdots+\right. \\
& \left.\quad+2 \int_{\substack{|x| \geqslant \sqrt{K / \gamma} \\
|y| \geqslant \sqrt{K / \gamma}}}^{\infty} \mathrm{d} x \mathrm{~d} y \tilde{\mathcal{R}}(x, y) \exp -\left[\frac{k_{\mathrm{B}} T}{4 \pi \gamma} q_{z}^{2} \log \left(\frac{\gamma}{K}\left(x^{2}+y^{2}\right)\right)\right]\right\} .
\end{aligned}
$$

The first integrai, of the order of $\Delta q_{x} \Delta q_{y} K / 4 \gamma$ is negligble Most generally, and in particular for our apparatus, the resolution is much better along one axis (here the $x$ axis, since $\Delta q_{x} \approx 12 \times 10^{5} \mathrm{~m}^{-1}, \Delta q_{y} \approx 6.8 \times 10^{8} \mathrm{~m}^{-1}$, see paragraph 21 ). It, follows that, in our case, the average properties of the interface are determined on an area extending over $\approx 15 \mathrm{~nm}$ along $y$, and over $\approx 10 \mu \mathrm{m}$ along $x$. Hence, the variation of the function along the $y$ axis over the domain ' integration can be neglected One obtains :

$$
R\left(q_{z}\right)=R_{\mathrm{F}}\left(q_{z}\right)\left(\frac{\gamma}{K}\right)^{-\frac{k_{\mathrm{B}} T}{4 \pi \gamma} q_{z}^{2}} 2 \int_{\sqrt{K / \gamma}}^{\infty} \frac{\delta q_{x}}{\sqrt{2 \pi}} \mathrm{e}^{-\frac{1}{2} \Delta q_{x}^{2} x^{2}} x^{-2 \frac{k_{\mathrm{B}} T}{4 \pi \gamma} q_{z}^{2}}
$$

which gives .

$$
R\left(q_{z}\right)=R_{\mathrm{F}}\left(q_{z}\right)\left(\frac{2 \gamma}{K \Delta q_{x}^{2}}\right)^{-\frac{k_{\mathrm{B}} T}{4 \pi \gamma} \dot{q}_{z}^{2}}\left[\frac{\Gamma\left(\frac{1}{2}-\frac{k_{\mathrm{B}} T}{4 \pi \gamma} q_{z}^{2}, \frac{1}{2} \Delta q_{x}^{2} \frac{K}{\gamma}\right)}{\sqrt{\pi}}\right]
$$


where $\Gamma(a, x)$ is the incomplete $\Gamma$ function Developing the $\Gamma$ function for $\left(k_{\mathrm{B}} T / 4 \pi \gamma\right) q_{z}^{2} \ll 1$, one obtains.

$$
R\left(q_{z}\right)=R_{\mathrm{F}}\left(q_{z}\right) \mathrm{e}^{-\left[\frac{k_{\mathrm{B}} T}{4 \pi \gamma} q_{z}^{2} \log \left(\frac{2 \gamma}{K \Delta q_{x}^{2}}\right)\right]}\left[1+(\gamma E+2 \log 2) \frac{k_{\mathrm{B}} T}{4 \pi \gamma} q_{z}^{2}\right]
$$

where $\gamma_{E}$ is Euler's constant, or :

$$
R\left(q_{z}\right) \approx R_{\mathrm{F}}\left(q_{z}\right) \mathrm{e}^{-\frac{k_{\mathrm{B}} T}{4 \pi \gamma} q_{x}^{2}\left[\log \left(\frac{2 \gamma}{K \Delta q_{x}^{2}}\right)-\left(\gamma_{\mathrm{E}}+2 \log 2\right)\right] .}
$$

which is of the form $[31,32]$

$$
R\left(q_{z}\right)=R_{\mathrm{F}}\left(q_{z}\right) \mathrm{e}^{-q_{z}^{2} \sigma^{2}}
$$

where the measured roughness $\sigma$ is such that

$$
\sigma^{2}=\frac{k_{\mathrm{B}} T}{4 \pi \gamma}\left[\log \left(\frac{\gamma}{K \Delta q_{x}^{2}}\right)-\left(\gamma_{\mathrm{E}}+\log 2\right)\right]
$$

For water and with $\Delta q_{x} \approx 12 \times 10^{5} \mathrm{~m}^{-1}$, one obtains $\sigma=0297 \mathrm{~nm}$, significantly smaller than the intrinsic value (A 4) This equation differs from reference [32] by the addition of corrective terms and without an additional parameter related to the atomic size.

\section{References}

[1] AdAm N K, "The Physics and Chemistry of Surfaces" 3rd edition (Oxford University Press, Oxford) 1941

[2] Harkins W D, "The Physical Chemistry of Surface films " (Rheinhold Publishing Corp, NewYork), 1952

[3] GAINES G L «Insoluble Monolayers at Liquid-Gas Interfaces " (Wiley Interscience, New-York) 1966

[4] Cadenhead D A., Muller-landau F and Kellner B $M$ J, in «Ordering in Two Dimensions " S K Sinha Ed (North Holland, Amsterdam) 1980, p 73

[5] Stallberg-Stenhagen S and Stenhagen E, Nature 156 (1945) 239.

[6] LundQuist M., Chemica Scripta 1 (1971) 5

[7] Albrecht O, Gruler H and Sackmann E, J Phys. France 39 (1978) 301.

[8] Georgallas A and Pink D A, Can J Phys 60 (1982) 1678

[9] Fischer A, Lösche M , Möhwald H and Sackmann E , $J$-Phys Lett France 45 (1984) L-785

[10] Pallas N R and Pethica B A, Langmuir 1 (1985) 509

[11] Winch P J and EARnshaw J C., J Phys Condens.-Matter 1 (1989) 7187.

[12] Miller A, KNoll W and Möhwald H, Phys Rev Lett 56 (1986) 2633

Moore B, KNobler C. M, Broseta D and Rondelez F, $J$ Chem Soc Faraday Trans. 82 (1986) 1753

[13] Bibo A M. and Peterson I R, Thin Sold Films 178 (1989) 81

[14] Abraham B $M$, Miyano $K$ and Ketterson J B , in "Ordering in Two Dimensions", $S \mathrm{~K}$ Sinha Ed (North Holland, Amsterdam) 1980, p 447

MiYano K and VeYsSIE M, Phys Rev Lett 52 (1984) 1318

MiYano K and MaEdA T., Rev Scl. Instrum 58 (1987) 428 
[15] HARD S and NeumanN R D, J Collold Interface Scı 83 (1981) 315, 120 (1987) 1

[16] Langevin D and Bouchiat M A., C R Acad Scl B 272 (1971) 1422

LANGEVIN D, $J$ Collotd Interface Sct 80 (1981) 412

[17] Earnshaw J C, MCGivern R. C. and Winch P J, J Phys. France 49 (1988) 1271

Earnshaw J C, MCGivern R C., MCLaughlin A C and Winch P J, Langmuir 6 (1990) 649

[18] Rasing Th., Shen Y R, Kim W M and Grubbs S, Phys Rev Lett. 55 (1985) 2903

KIM M W, RAsing Th. and Shen Y R, in "Phase Transitions in Soft Condensed Matter ", T Riste and D Sherrington Ed (Plenum Press, New-York) 1989

[19] Miller A, KNoll W and Möhwald H, Phys Rev. Lett 56 (1986) 2633

[20] Kjaer K., Als-Nielsen J , Helm C A, LaXhuber L A and Möhwald H, Phys. Rev Lett 58 (1987) 2224.

Dutta P, Peng J B, Lin B, Ketterson J B, Prakash M, Georgeopoulos P and EHRLICH S., Phys Rev Lett. 58 (1987) 2228

Wolf S, Leiserowitz L, Lahav M , Deutsch M , KJaer K and Als-Nielsen J , Nature 328 (1987) 63

Barton S, Thomas B, Flom E, Rice S A, Lin B., Peng J B and Ketterson J B , $J$ Chem Phys 89 (1988) 2257.

Lin B, Peng J. B , Ketterson J, B, Dutta P, Thomas B., Buontempo J and Rice S A., $J$ Chem Phys 90 (1989) 2393

[21] Kjaer K, Als-Nielsen J, Helm C A, Tippmann-Krayer P and Mohwald H., $J$ Phys. Chem 93 (1989) 3200

[22] Lin B, Shih M C., Bohanon T M, ICe G. E and Dutta P, Phys. Rev Lett 65 (1990) 191

[23] Bosio L, Benattar J J. and Rieutord F., Revue Phys. Appl 22 (1987) 775

RICHARDSON R M. and ROSER S J, Liquid Crystals 2 (1987) 797.

Helm C A, Mówald H, KJaer K and Als-Nielsen J, Europhys. Lett 4 (1987) 697

[24] Daillant J, Bosio L, Benattar J J and Meunier J, Europhys Lett 8 (1989) 453

[25] Daillant J , Bosio L and Benattar J J., Europhys Lett 12 (1990) 715

[26] Buhaenko M R., Grundy M J, Richardson R. M. and Roser S J, Thin Solid Films 159 (1988) 253

[27] JAmes R W , «The Optical Principles of the Diffraction of X-rays » Bell (London, 1948) p 135

[28] OXTOBY D W, Novak F and Rice S A, J Chem Phys 76 (1982) 5278

[29] BORN M and WOLF E., "Principles of Optics » 6th edition (Pergamon, London) 1980, p 491

[30] Jackson J D., "Classical Electrodynamics ", 2nd edition (Wiley, New-York) 1975, p. 418.

GABOR D, Rev Modern Phys 28 (1956) 260

[31] WU E S. and WebB W W, Phys Rev A 8 (1973) 2065

[32] Braslau A, Pershan P S, Swislow G, Ocko B M and Als-Nielsen J, Phys Rev. A 38 (1988) 2457

[33] Guggenheim E. A , Thermodynamics an Advanced Treatment for Chemists and Physicists » (North Holland, Amsterdam) 1967

[34] Grundy M J, Richardson R M, Roser S. J, Penfold J. and Ward R C, Thin Solid Films 159 (1988) 43

[35] Bosio L, Cortes R, Folcher G. and Oumezine M, Revue Phys Appl 20 (1985) 437

[36] Middleton S R, Iwahashi M, Pallas N R and Pethica B A, Proc. $R$ Soc Lond. $A 396$ (1984) 143.

[37] Inahashi M., Maehara N, Kaneko Y, Seimiya T, Middleton S R, Pallas N R and Pethica B A, $J$ Chem. Soc Faraday Trans 81 (1985) 973

[38] Braslau A, Deutsch M, Pershan P S, Weiss A H, Als-Nielsen J. and Bohr J, Phys Rev Lett 54 (1985) 114

[39] Meunier J, J Phys France 48 (1987) 1819

[40] Szleifer I, Ben-Shaul A and Gelbart W M, J Phys Chem 94 (1990) 5081

[41] Halperin A, Alexander S, Schechter I, J. Chem Phys. 86 (1987) 6550

Chen Z Y, Talbot J., Gelbart W and Ben-Shaul A., Phys Rey Lett. 61 (1988) 1376

[42] Bareman J P, Cardini G and Klein M L., Phys Rev Lett 60 (1988) 2152. 
[43] IsRaelashvili J N, «Intermolecular and Surface Forces » (Academic Press, London) 1985, p 249

[44] KaTO T, Langmuir 6 (1990) 870

[45] Als-Nielsen J. and Móhwald H., to appear in' « Handbook of Synchrotron Radiation " (1989)

[46] Beaglehole D, Physica 100B (1980) 163

[47] LeKner J , Physica 103 (1982) 544

[48] MEunier J, $J$ Phys France 46 (1085) L-1014

[49] Brochard F and LenNon J F, J Phys France 36 (1975) 1036

[50] Servuss R M., Harbich W and Helfrich W, Biochem Biophys Acta 436 (1976) 900

[51] Engelhardt H, Duwe H. P and Sackmann E, J Phys France 46 (1985) 1395.

Faucon J F, Mitov M D, Méléard P, BIVAs I and Bothorel P, $J$ Phys France, 50 (1989) 2389 , and references therein

[52] Evans E A, Biophys $J 43$ (1983) 27

[53] Di Meglio J M, Dvolaitzky M and Taupin C., $J$ Phys Chem 89 (1985) 871

[54] Safinya C R, Sirota E. B, Roux D and SMITH G S, Phys Rev Lett 62 (1989) 1134

[55] Di Meglio J M, Dvolaitzky M, Léger L. and Taupin C, Phys Rev Lett 54 (1985) 1686

[56] Szleifer I, Kramer D, Ben-Shaul A, RouX D and Gelbart W M, Phys Rev Lett 60 (1988) 1966

Ben-Shaul A., Szleifer I and Gelbart W M, in « Physics of Amphiphilıc layers » J Meuner,

- D. Langevın and N Boccara Eds (Springer, Berlın) 1988 ,

[57]-Milner S T and Witten T A, $J$ Phys. France 49 (1988) 1951.

[58] Landau L D and Lifschitz E M., "Theory of Elasticity (Pergamon Press, London) 1970, p 44

[59] Adin MANN J Jr, Langmutr 1 (1985) 10.

[60] Daillant J, Benattar J J and Bosio L, to appear in $J$ Phys Condensed Matter

[61] Peliti L and Prost J, J. Phys France 50 (1989) 1557

[62] Fogden A, Mitchell D J. and Nimham B. W, Langmuir 6 (1990) 159.

[63] Petrov A G and Bivas I, Progr Surface Scl 16 (1984) 389.

[64] Goddard E. D, SMITH S R and KaO O, J. Collozd Interf Sci 21 (1966) 320

[65] Beckmann P and Spizzichino A, "The Scattering of Electromagnetic Waves from Rough Surfaces» (Pergamon Press, London) 1963

MCGINN A and SYKes J, Harwell Report AERE-R-9768

[66] Croce P, J . Opt (Paris) 11 (1980) 243, 14 (1983) 213; 15 (1984) 243

[67] Sinha S K., Sirota E B, Garoff S and Stanley H. B, Phys Rev. B 38 (1988) 2297 